\title{
3814
}

\section{The Development of the Quality of Life Impact of Refractive Correction (QIRC) Questionnaire.}

\author{
K. Pesudovs ${ }^{1}$, D.B. Elliott ${ }^{1}$, S.D. Gelsthorpe ${ }^{2}$, E. Garamendi ${ }^{1}$, J.M. Burr ${ }^{3}$. \\ 1. Department of Optometry, \\ 2. School of Health Studies, University of Bradford, Bradford, United Kingdom. \\ 3. Department of Ophthalmology, Leeds General Infirmary, Leeds, United Kingdom.
}

\section{Purpose}

- To develop an instrument for the comprehensive measurement of the impact of refractive correction on quality of life in the pre-presbyopic age group.

- To design the questionnaire to be relevant to refractive correction by spectacles, contact lenses and refractive surgery such that it is suitable for comparison between correction mode and for studies of treatment outcome.

\section{Background}

- The measurement of quality of life has become an essential component of outcomes assessment.

- At the most fundamental level questionnaire-based quality of life assessment represents a standardisation of the clinical practice of gaining a subjective evaluation of treatment (or disease) impact from the patient.

- This is especially relevant to refractive outcome because of the difficulties of measuring subtleties of visual outcome for refractive surgery or other refractive correction. 


\section{Methods}

To ensure construct validity ${ }^{1}$ the following steps for questionnaire development were taken:

- Domain selection

- Item selection

- Initial item reduction

- Question scaling

- Pilot questionnaire data collection

- Further item reduction (Basic statistics, Factor analysis and Rasch scaling)

\section{Domain selection - method}

The quality of life literature was reviewed to identify all domains of quality of life.

- These were discussed in a focus group of eye care and psychology professionals for reduction to those thought to be influenced by refractive correction.

\section{Domain selection - result}

- Seven domains of quality of life were selected:

Visual function, Symptoms, Convenience, Work / Economic issues, Health Concerns, Interaction with Other Senses, Feelings (well-being)

\section{Item selection - method}

- Items within these domains were selected from the scientific literature and from suggestions of professionals and lay people.

- The literature search included global quality of life instruments and those specific to vision and cosmetic surgery. ${ }^{2,3}$

- Professionals included 63 clinicians and clinical staff working in optometry, contact lens fitting, optical dispensing, ophthalmology, refractive surgery and psychology.

- Lay people included spectacle wearers, contact lens wearers and people who have had refractive surgery. 


\section{Item selection - result}

- 647 items were selected as possibly being relevant:

Visual function 176, Feelings 192, Convenience 85, Symptoms 97, Work/Economic issues 54, Health concerns 19, Other senses 24).

\section{Initial item reduction - method}

These 647 items were likely to include many issues that were only relevant to a minority of people. Such issues cannot be included in the final questionnaire as they will result in a high proportion of missing data and therefore would not be statistically contributory to an overall measure.

Focus groups were used to reduce the 647 items down to only those likely to be relevant to a majority of people.

- 3 professional focus groups (psychology and eye care professionals experienced with spectacle, contact lens and refractive surgery patients) did the initial reduction.

- 3 lay public focus groups (one group of each correction mode) were presented with a mock questionnaire including this reduced set of questions.

Initial item reduction - result

- $\quad$ The 3 professional focus groups reduced the number of items 115 .

- $\quad$ The 3 lay focus groups further reduced the number of items to 90 .

\section{Question scaling - method}

- A variety of prototype response scales were chosen following a review of the questionnaire design literature.

- These were presented to the lay focus groups in the mock questionnaire for feedback.

\section{Question scaling - result}

- A five point response scale with descriptors chosen that were likely to be interpreted as evenly spaced. e.g.

Don't know / Not at all A little Moderately Quite a lot Extremely Not applicable 


\section{Pilot questionnaire data collection}

- The pilot questionnaire was constructed from the 90 items and the response scales arrived at via the aforementioned steps.

- The questionnaire was completed by 306 subjects (102 spectacle wearers, contact lens wearers and people who have had refractive surgery).

- Information from equal numbers of people using the three correction modes was obtained to minimise bias in favour of any correction type.

- The three groups were similar to national population norms for each mode of correction in terms of age, gender, race and socio-economic status.

\section{Item reduction using pilot data (Basic statistics - method)}

- Questions with a large proportion (>33\%) of missing data were not sufficiently contributory and were therefore removed.

- Questions with non-normal distributions were not suitable for further (factor) analysis. Thus, questions with skew or kurtosis values outside the range 2.00 to +2.00 , or questions with $95 \%$ of the data within 2 end responses were removed.

\section{Item reduction using pilot data (Basic statistics - result)}

- 31 questions eliminated

\section{Item reduction using pilot data (Factor analysis - method)}

Factor analysis was used to determine whether all items were measuring an overall concept (quality of life) and whether items could be grouped into subsections of this overall concept.

- A Principal components factor analysis with Varimax rotation was used.

Item reduction using pilot data (Factor analysis - result)

- Four major factors were established (> 7 items) with numerous minor factors (< 7 items): Convenience 15 items, Near - vision and symptoms 12 items, Work / Economic issues 8 items, Feelings (appearance) 7 items, Health concerns 6 items, Distance vision 5 items, Feelings (social) 3 items etc.

- No items were excluded, although 6 pairs of highly correlated ( $r>0.70$; implies redundant) items were noted for removal during Rasch scaling. 


\section{Item reduction using pilot data (Rasch scaling - method)}

- Rasch scaling was used to determine the validity of each question in terms of its fit to each subscale and the overall scale, determine the validity of the response scale, identify redundancy (allowing further item reduction), and weighting question responses to create a continuous scale. ${ }^{4}$

- Items were eliminated as redundant if the Infit mean square was $<0.80$ or if overlap was identified on the kidmap. Subscale numbers were minimised to retain a Cronbach's alpha $>0.80$.

Item reduction using pilot data (Rasch scaling - result)

- The response scale was reduced to 4 choices to improve fit.

- Valid subscales were identified for Convenience (8 items), Near vision and symptoms (5 items), Work / economic issues ( 6 items) and feelings (4 items).

- The overall scale was reduced to 29 items.

\section{Conclusions}

- Initial analyses have produced a 29-item questionnaire.

- This methodological approach for item selection, item reduction, question construction and response scaling ensures construct validity.

- Further testing of the reliability and validity of the questionnaire is required.

\section{References}

1. Jaeschke R, Guyatt GH. How to develop and validate a new quality of life instrument. In: Spilker B, ed. Quality of Life Assessment in Clinical Trials. New York: Raven Press, 1990:47-57.

2. Massof RW, Rubin GS. Visual function assessment questionnaires. Surv Ophthalmol 2001;45:531-48.

3. Fitzpatrick R, Jenkinson C, Klassen A, Goodacre T. Methods of assessing health-related quality of life outcomes for plastic surgery. Br J Plast Surg 1999;52:251-5.

4. Bond TG, Fox, CM. Applying the Rasch Model: Fundamental Measurement in the Human Sciences. Mahwah: Lawrence Erlbaum Associates, 2001.

\section{Acknowledgements}

- This project is funded by Johnson and Johnson Vision Care, Europe.

- K. Pesudovs is supported by NHMRC Sir Neil Hamilton Fairley Fellowship 007161. 